\title{
QUALITY DETERIORATION OF MASS REARED OFFSPRINGS OF Bracon hebetor IN SUCCESSIVE GENERATIONS
}

\author{
M. S. Alam* ${ }^{1}$, M. Z. Alam, ${ }^{2}$ S. N. Alam ${ }^{3}$, M. R. U. Miah, ${ }^{2}$ \\ M. I. H. Mian ${ }^{2}$ and M. M. Hossain ${ }^{2}$ \\ Entomology Division, Bangladesh Agricultural Research Institute, Gazipur, Bangladesh
}

\begin{abstract}
A study was undertaken to determine the generation-wise parasitizing efficiency of mass reared Bracon hebetor on wax moth (Galleria mellonella). Two experiments were conducted to achieve the objectives. In the first experiment, the host larvae and parasitoid were released in the field micro plot during first generation and reared in the laboratory in subsequent generations. In another experiment, all generations were developed in the laboratory. In the first experiment, generation showed significant variation on sex ratio of adult progeny production. The male sex ratio increased with the range of 62.89 to $74.58 \%$ and female sex ratio declined ranging from 39 to $25 \%$ with the advancement of generations. The percent parasitization and adult progeny production was not influenced appreciably with the progress of generation. In the second experiment parasitization and adult progeny production did not vary significantly in successive generations. However there was a decreasing trend in parasitization from 95 to $55 \%$ by Bracon hebetor and adult progeny production by 47.5 to 15.5 adultsfemale ${ }^{-1} 10$ larvae $^{-1}$ over generation. The sex ratio of adult progeny was significantly influenced by inbreed generation in laboratory reared population. Female decrease in sex ratio from first to sixth generation was 39.25 to $16.88 \%$.
\end{abstract}

Keywords: Bracon hebetor, inbreeding, quality, fitness

\section{INTRODUCTION}

Bracon hebetor Say (Hymenoptera: Braconidae) is an efficient gregarious larval parasitoid of many lepidopteran storage and vegetable pests. Artificial release

\footnotetext{
* Corresponding author email: alamvpd@gmail.com

${ }^{1}$ Vertebrate Pest Division, Bangladesh Agricultural Research Institute, Gazipur, Bangladesh

${ }^{2}$ Bangabandhu Sheikh Mujibur Rahman Agricultural University, Gazipur, Bangladesh

${ }^{3}$ Entomology Division, Bangladesh Agricultural Research Institute, Gazipur, Bangladesh
} 
of this parasitoid has the ability to control many devastating pests like Leucinodes orbonalis, Spodoptera sp., Helicoverpa armigera, Maruca vitrata, Corcyra cephalonica etc (Thanavendan and Jeyarani, 2010; Nikim and Pawar, 1993; AmirMaafi and Chi, 2006). Genetic variation in Hymenoptera reduced genetic variability due to the production of diploid males from fertilized eggs. At least two mechanisms of sex determination occur among Hymenoptera. Whiting (1943) proposed singlelocus complementary sex determination (sl-CSD) based on his studies of Bracon sp. Sex is determined by multiple alleles at a single locus, homozygosity leads to males and heterozygosity to females. All Hymenoptera (over 200000 species of ants, bees and wasps) are haplodiploid. Arrhenotoky is the most prevalent mode of reproduction, i.e. males develop parthenogenetically from unfertilized haploid eggs while females develop from fertilized diploid eggs (Cook, 1993; Cook and Crozier, 1995; Beukeboom et al., 2000 and Salin et al., 2004).

Complementary sex determination (CSD) is detected through inbreeding by mother-son and brother-sister mating. Homozygous diploid males can arise through inbreeding. Diploid male production can represent a significant load since diploid males are usually sterile (Cook and Crozier, 1995; Henshaw et al., 2002) and often non-viable (Petters and Mettus, 1980). Presence of diploid males is strongly correlated with inbreeding and constitutes a genetic load for the population as it results in a male biased sex-ratio \{(females mated to diploid males, like unmated females, produce only male offspring) Cook, 1993\}.

Most studies on $B$. hebetor indicated that wasps produce a female-biased sex ratio (Benson, 1973; Rotary and Gerling, 1973; Taylor, 1988; Strand and Godfray, 1989). Local mate competition is an important factor leading to female-biased sex ratios in other species of gregarious parasitoids and is an obvious candidate to explain the basis found in $B$. hebetor. However, in species with this type of sex determination was found in $B$. hebetor, mating between sibling (mother-son and brother-sister mating) leads to the production of diploid males and a substantial drop in fitness (Galloway and Grant, 1989).

In North American B. hebetor, few diploid males survived to become adults and of these around 90\% were sterile (Whiting, 1925; Torvik, 1931). This sterility was caused by an inability of most diploid sperm to penetrate the eggs (MacBride, 1946). Occasionally some sperm successfully fertilized an egg, but the resulting triploid females were also sterile (Torvik, 1931). In B. hebetor, a much smaller percentage (20-40\%) of diploid males was sterile. Diploid males of Habrobracon serinopae (Cherian) males did not have a higher mortality, but their fertility was unknown (Petters \& Mettus, 1980). Henter (2003) showed that an outbreeding haplodiploid wasp Uscana semifumipennis (Hymenoptera: Trichogrammatidae) suffers substantial inbreeding depression. Longevity was $38 \%$ shorter, fecundity was $32 \%$ lower, and sex ratio was $5 \%$ more male for experimentally inbred wasps when compared to outbreed controls. Zhou et al. (2007) studied the three fitness traits (i.e., 
development time, brood size and body size), as well as sex ratio, between experimentally imposed inbred and outbred populations on Cotesia glomerata, a gregarious parasitoid of diamond back moth. Results recorded by other investigator show that one generation of full-sib mating in C. glomerata resulted a significantly higher proportion of males $(43 \%)$ compared to outbred control $(29 \%)$, and the sex ratio shifts are largely in agreement with sl-CSD (single locused complementary sex determination) model (Petters and Mettus, 1980).

However, the parasitizing efficiency of mass reared parasitoid in the laboratory may be decreased, so it is important to determine the generation wise parasitizing efficiency of mass reared $B$. hebetor to maintain the quality of the laboratory population for effective biological control program. Considering the above facts the present study was undertaken

\section{MATERIALS AND METHODS}

The study was undertaken in the micro plot of brinjal field and in the laboratory of Entomology Division, BARI, Gazipur during February 2011 to July 2011. Two experiments were conducted.

\section{Parasitization of host larvae in the field and subsequently in the laboratory}

Ten host larvae of wax moth, Galleria mellonella were released on the plant in the caged micro plot $(1.5 \mathrm{~m} \mathrm{x} 1 \mathrm{~m})$ of brinjal field. Three brinjal plants were grown in each micro plot. The plants were then encircled with 32 mesh nylon net. Ten Bracon hebetor adult ( 6 female and 4 male) were then released on the plant in the caged micro plot. After 2-3 days the parasitized larvae were collected from the field and kept in the petridishes. The numbers of parasitoid adults were recorded and kept in test tube $(10 \mathrm{~cm} \times 2 \mathrm{~cm})$ containing honey solution. The successive generations (up to 7 generations) were continued in the laboratory. Ten last instars (5-6 instar) wax moth, Galleria mellonella L. larvae were released in a plastic jar $(1000 \mathrm{ml})$ having a corrugated paper sheet. The full-fed larvae took position on the corrugated paper sheet for pupation. Two B. hebetor adults (1 male and 1 female) emerged from field collected paralyzed G. mellonella were released in the plastic jar with honey solution on the cotton ball for their food. The mouth of the plastic jar was closed with black cloth. The plastic jars with wax moth larvae and $B$. hebetor were kept on a rack in the laboratory for 8-10 days for parasitization, egg laying, pupation and emergence of adult $B$. hebetor. The experiment was conducted four times. Data on generation-wise

parasitization, total adults emergence per female and sex ratio were recorded carefully.

\section{Parasitization of host larvae in the laboratory}

Ten last instars (5-6 instar) wax moth, Galleria mellonella L. larvae were released in a plastic jar $(1000 \mathrm{ml})$ having a corrugated paper sheet inside. The full-fed larvae took position on the corrugated paper sheet within the plastic jar for pupation. 
One pair laboratory reared adults of $B$. hebetor ( 1 male and 1 female) were released in the plastic jar having honey solution soaked in cotton ball for their food. The mouth of the jar was closed with black cloth. The jar containing wax moth larvae and $B$. hebetor were placed on a rack in the laboratory for 8-10 days for parasitization, egg laying, pupation and adult emergence of parasitoid. The experiment was continued for successive 6 generations. Data on generation-wise parasitization, total adults per female and sex ratio were recorded.

\section{Data analysis}

Data on parasitism, offspring production and sex ratio were analyzed statistically using one-way analysis of variance (ANOVA) following SPSS 16 software and means were compared following Tukey,s Honestly Significant Different Test. The percent data were transformed to arcsine before analysis. Relationship of percent parasitization, adult progeny production and sex ratio with generation were measured by regression analysis.

\section{RESULTS}

In the first experiment; percent parasitization by $B$. hebetor at first generation collected from the caged micro plot on the host ( $G$. mellonella) larvae did not vary significantly with the progress of generations (Figure 1). However, parasitization efficiency was more or less similar up to $5^{\text {th }}$ generation $(80 \%)$ and decreased sharply from 6th generation showing the lowest parasitization at $6^{\text {th }}$ generations. Similarly progeny production was not varied significantly with generations (Figure 2). The total adult progeny production was maximum at generation $5^{\text {th }}$ (54 adult female ${ }^{-1} 10$ $\left.\operatorname{larvae}^{-1}\right)$ generation followed by $4^{\text {th }}\left(47.25\right.$ adults female ${ }^{-1} 10$ larvae $\left.^{-1}\right), 1^{\text {st }}(39.75$ adults female $^{-1} 10$ larvae $\left.^{-1}\right)$ and $7^{\text {th }}\left(38.75\right.$ adults female ${ }^{-1} 10$ larvae $\left.^{-1}\right)$ generations. The lowest adult progeny production was recorded in generation $6^{\text {th }}\left(30.25\right.$ adults female ${ }^{-1}$ 10 larvae $^{-1}$ ) (Figure 2).

Generation showed considerable influence of sex ratio of adult progeny production. The proportion of male increased gradually with the advancement of generation. On the other hand a decreasing trend was observed in female sex ratio with advancement of generation within the range of 39 to $25 \%$ (Figure 3). The relationship of male sex ratio $(r=0.785)$ was positive and female sex ratio $(r=-$ 0.798) was negative with generations (Figure 4).

In the second experiment; generation wise parasitization by $B$. hebetor reared in laboratory ranged between 55 to $95 \%$. Significantly highest percent parasitization was recorded from first generation (95\%) followed by third (80\%) and fourth $(72 \%)$ generations. The lowest parasitization was found in sixth generation $(55 \%)$ followed by fifth and second generations. Difference in parasitization at second, third and fourth generations were not significant. Similarly, the parasitization at third, fourth and fifth generation was statistically similar. The parasitization decreased with the progress of generation. Their relationship was linear, negative significant $(r=-$ 
0.841). The $\mathrm{R}^{2}$ values (0.712) indicate that influence of generation on the variation of parasitization is $71.20 \%$ (Figure 5).

Total adult progeny production ranged 15.5-47.5 female $^{-1}$ from firs to sixth generations. The trend in total adult progeny production was more or less similar to parasitism by $B$. hebetor. The first generation produced 47.50 adult female ${ }^{-1} 10$ larvae $e^{-1}$, which was statistically similar to 2 nd $\left(42.25\right.$ adults female ${ }^{-1} 10$ larvae $\left.^{-1}\right)$ and 3rd (32.75 adults female ${ }^{-1} 10$ larvae $\left.^{-1}\right)$ generations. The lowest adult progeny (15.5 adults female ${ }^{-1} 10$ larvae $^{-1}$ ) recorded at 6th generation, which was statistically similar to 4 th and 5th generations. The relationship between total adult progeny production ( $\mathrm{r}$ $=-0.96$ ) and generation was linear, negative and significant (Figure 6).

Prevalence of male and female at first (F1) to sixth generation (F6) ranged from 67.75-83.12 and $16.88-39.25 \%$ among adult progeny produced per 10 host larvae, respectively. The highest and lowest percentages of male and lowest and highest percentage of female were recorded from F6 and F1, respectively. However, the percentage of male was higher compared to male at every generation. Regression analysis showed that relationship of male population $(\mathrm{r}=0.976)$ was positively and that of female was negatively correlated $(\mathrm{r}=-0.97)$ with sib mating generation (Figure 7).

\section{DISCUSSIONS}

Results of the present study revealed that population of male increased and that of female decreased gradually and considerably with the progress of generations of Bracon hebetor. At each generation, prevalence of male was considerably higher than that of female. Similar results have been reported by many other investigators. Henter (2003) studied the inbreeding depression in solitary parasitoid tricogrammatids wasp, Uscana semifumipennis, which suffers it substantially. Longevity was $38 \%$ shorter, fecundity was $32 \%$ lower and sex ratio was 5\% more male for experimentally inbred wasp when compared to outbreed control. In another study, inbreeding lead to an increase in the proportion of male offspring due to the production of diploid sons. This study was done on a gregarious parasitoid, Cotesia glomerata by comparing three fitness traits (i.e., development time, brood size and body size, as well as sex ratio) between inbred and outbreed population (Henter, 2003).

Results showed that one generation of full sib mating in C. glomerata resulted in a significantly higher proportion of male (43\%) compared with outbreed control $(29 \%)$ and sex ratio shifts are largely in agreement with sl-CSD model. When inbred level increased over four generation brood sex ratios become male-biased (70\%) (Zhou et al., 2007). Sibling mating increases the production of males, leading to the male-biased sex ratio of broods in the laboratory which was responsible for sl-CSD mechanism (Zhou et al., 2006). Braconid wasp Bracon hebetor; a gregarious parasitoid in which sl-CSD was first described by whiting (1943). In single-locus sex determination (sl-CSD), the sex of an individual is determined by alleles at a single 
sex locus. Diploid individuals that are heterozygous at the sex locus develop into females, individuals that are hemizygous or homozygous at the sex locus become males (Whiting, 1943). Since in most cases diploid males are inviable or sterile, their production imposes a genetic load on the population. sl-CSD is considered to be incompatible with inbreeding as it will enhance the chance of homozygosity at the sex determination locus, and thus lead to the increased production of diploid males (Bruckner, 1978; Cook and Crozier, 1995). The production of diploid males under inbreeding has been suggested to be partly responsible for the failures in biological control with Ichneumonidae and Braconidae species (Stouthamer et al., 1992).

Single-locus sex determination substantially influences the sex ratio and the population growth of parasitoid species. Both factors affect the biological control potential of parasitoids. Clearly, single-locus sex determination has the potential to impede biological control severely if appropriate precautions are not taken (Cook, 1993; Zhou and Dorn, 2007; Heimpel and de Boer, 2008; Elias et al., 2010). Southamer et al. (1992) suggested two approaches to maximize the diversity of different sex alleles during laboratory propagation and mass rearing: (1) the wasps can be maintained as one large population, or (2) they can be kept as a large number of isolated subpopulations. The first approach reduces the rate at which alleles are lost. The second approach results in many alleles being lost in each subpopulation, but at least two sex alleles are retained.

\section{CONCLUSION}

There was a decreasing trend in parasitization from 95 to $55 \%$ by $B$. hebetor and adult progeny production by 47.5 to 15.5 adultsfemale $^{-1} 10$ larvae $^{-1}$ over generation in laboratory reared population. The sex ratio of adult progeny had been significantly influenced by inbreed generation in laboratory reared population. Female decrease in sex ratio from first to sixth generation was 39.25 to $16.88 \%$.

\section{ACKNOWLEDGEMENTS}

This is a part of $\mathrm{Ph}$. D work. This work has been financially supported by the Bangladesh Agricultural Research Institute strengthening fund phase-2, Ministry of Agriculture, The Government of the Peoples Republic of Bangladesh. The author is grateful to financial authority.

\section{REFERENCES}

Amir-Maafi, M and Chi, H. 2006. Demography of Habrobracon hebetor (Hymenoptera: Braconidae) on Two Pyralid Hosts (Lepidoptera: Pyralidae). Annals Entomological Society of America, 99(1): 84-90

Benson, J. F. 1973. Intraspecific competition in the population dynamics of Bracon hebetor Say. Journal of Animal Ecology, 42:193-197 
Beukeboom, L. W., Ellers, J. and Van Alphen, J. J. M. 2000. Absence of single locus complementary sex determination in the braconid wasps Asobara tabida and Alysia manducator. Heredity, 84: 29-36

Bruckner, D. 1978. Why are there inbreeding effects in haplodiploid system? Evolution, 32: 456-458

Cook, J. M. and Crozier. R. H. 1995. Sex determination and population biology in the Hymenoptera. Trends Ecological Evolution, 10: 281-286

Cook, J. M. 1993. Sex determination in the Hymenoptera: A review of models and evidence. Heredity, 71: 421-435

Elias, J., Dorn, S., Mazzi, D. 2010b. Inbreeding in a natural population of the gregarious parasitoid wasp Cotesia glomerata. Molecular Ecology, 19: 2336-2345

Galloway, K. S., Grant, B. 1989. Reverse sex ratio adjustment in an apparently outbreeding wasp, Bracon hebetor. Evolution, 43: 465-468

Heimpel, G. E., de Boer, J. G. 2008. Sex Determination in the Hymenoptera. Annual Review of Entomology, 53:209-230

Henshaw, M. T, Queller, D. C. and Strassmann, J. E. 2002. Control of male production in the swarm-founding wasp, Polybioides tabidus. Journal of Evolution Biology, 15: 262-268

Henter, H. J. 2003. Inbreeding depression and haplodiploidy: experimental measures in a parasitoid and comparisons across diploid and haploid insect taxa. Evolution, 57(8): $1793-1803$

MacBride, D. H. 1946. Failure of sperm of Habrobracon diploid males to penetrate the eggs. Genetics, 31: 224

Nikim, P. K. and Powar, C. V. 1993. Life tables and intrinsic rate of natural increase of Bracon hebetor (Say)(Hymenoptera: Braconidae) population on Corcyra cephalonica (Staint)(Lepidoptera: Pyralidae), a key parasitoid of Helicoverpa armigera (hubner) (Lepidoptera: Noctuidae). Journal of Applied Entomology, 115(2): 210-213

Petters, R. M. and Mettus, R. V. 1980. Decreased diploid male viability in the parasitic wasp, Bracon hebetor. Journal of Heredity, 71:353-356

Rotary, N. and Gerling, D. 1973. The influence of some external factors upon the sex ratio of Bracon hebetor Say (Hymenoptera: Braconidae). Environmental Entomology, 2: 134138

Salin. C., Deprez, B., Van Bockstaele, D. R., Mahillon, J., Hance, T. 2004. Sex determination mechanism in the hymenopteran parasitoid Aphidius rhopalosiphi De Stefani-Peres (Braconidae : Aphidiinae). Belgium Journal of Zoology, 134 (2/1): 15-21

Stouthamer, R., Luck, R. F. and Werren, J. H. 1992. Genetics of sex determination and the improvement of biological control using parasitoids. Environmental Entomology, 21: 427-435

Strand, M. R. and Godfray, H. C. J. 1989. Superparasitism and ovicide in parasitic Hymenoptera: theory and a case study of the ectoparasitoid Bracon hebetor. Behavior of Ecological Sociobiology, 24: 421-432 
Taylor, A. D. 1988. Host effects on larval competition in the gregarious parasitoid Bracon hebetor. Journal of Animal Ecology, 57: 163-172

Thanavendan, G. and Jeyarani, S. 2010. Effect of different temperature regimes on the biology of Bracon brevicornis Wesmael (Braconidae: Hymenoptera) on different host larvae. Journal of Bio-pesticide, 3(2): 441-444

Torvik, M. M. 1931. Genetic evidence for diploid is in of biparental males in Habrobracon. Biological Bulletin, 61: 139-156

Whiting, A. R. 1925. The inheritance of sterility and of other defects induced by abnormal fertilization in the parasitic wasp, Habrobracon juglandis. Genetics, 10: 33-58

Whiting, P. W. 1943. Multiple alleles complementary sex determination of Habrobracon. Genetics, 28: 365-382

Zhou, Y., Gu, H. and Dorn, S. 2007. Effects of inbreeding on fitness components of Cotesia glomerata, a parasitoid wasp with single-locus complementary sex determination (slCSD). Biological Control, 40:273-279

Zhou, Y., Gu, H. and Dorn, S. 2006. Single-locus sex determination in the parasitoid wasp Cotesia glomerata (Hymenoptera: Braconidae). Heredity, 96: 487-492 


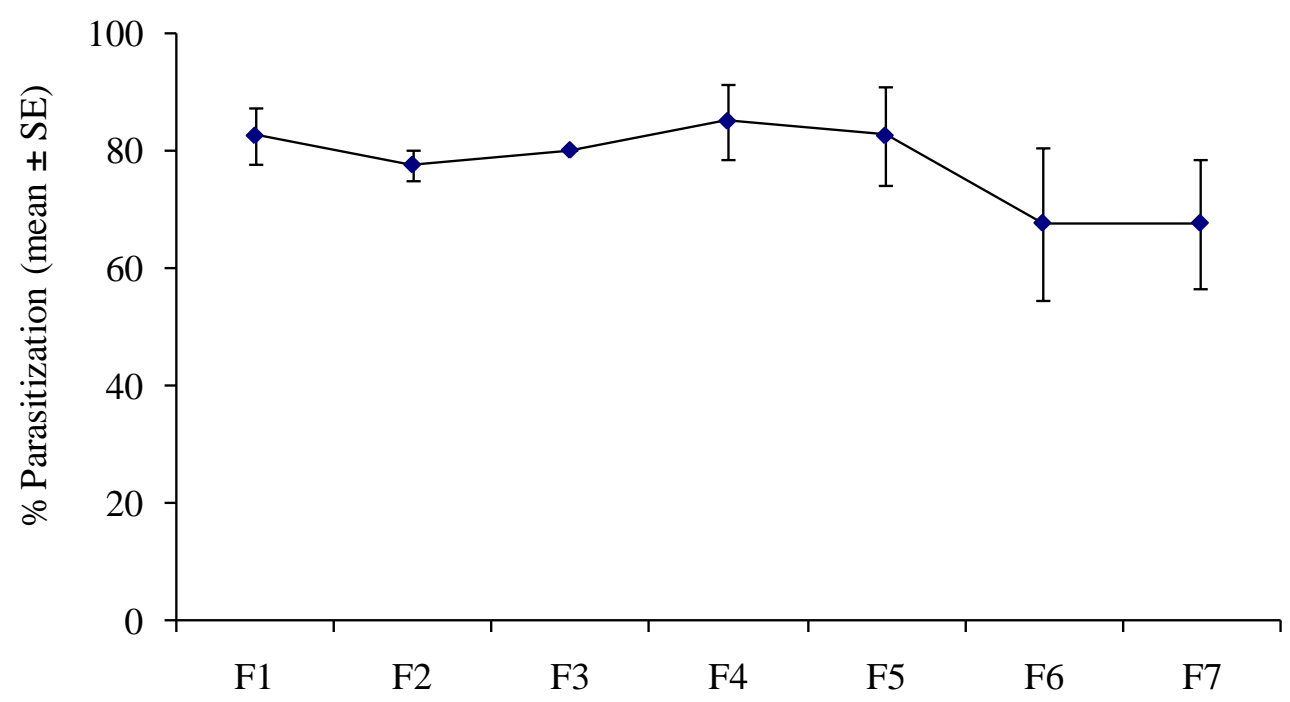

Generation

Figure 1. Generation wise mean parasirism (\%) by Bracon hebetor in first generation parasitism in the caged micro plot in the field and the successive generations in the laboratory. Vertical line in line graph show standard error of mean.

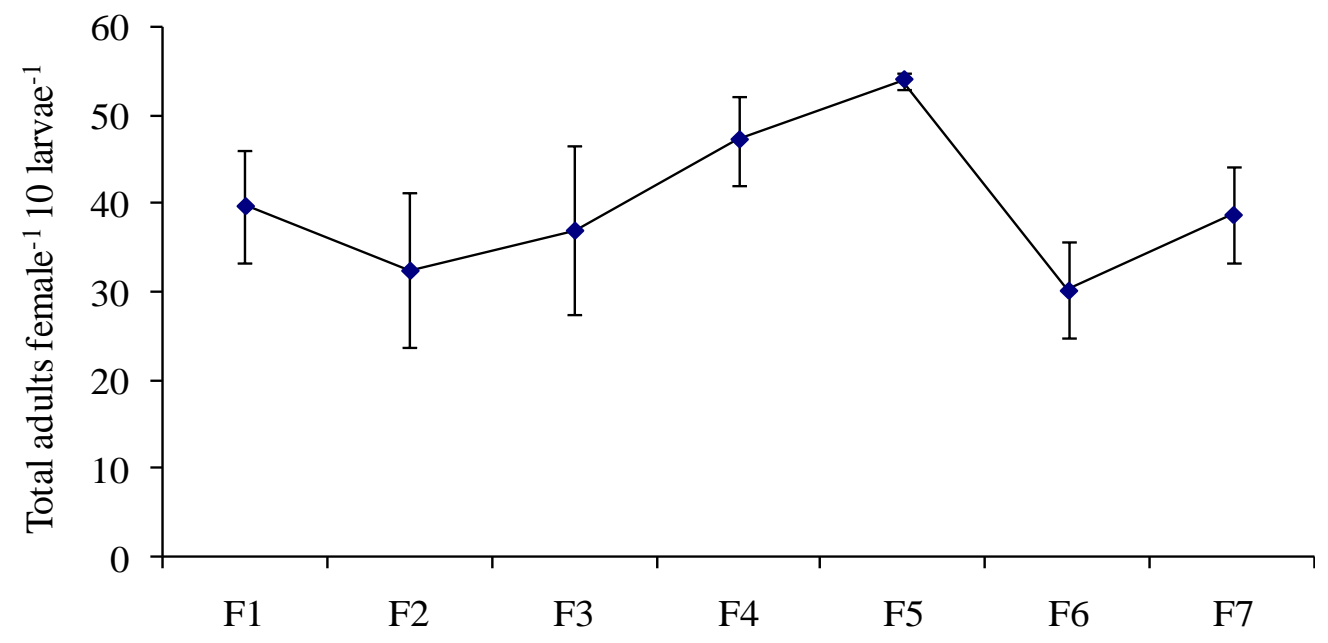

Generation

Figure 2. Generation wise mean number of offspring per female of Bracon hebetor in first generation in micro plot in the field and the successive generations in the laboratory. Vertical line in line graph show standard error of mean. 


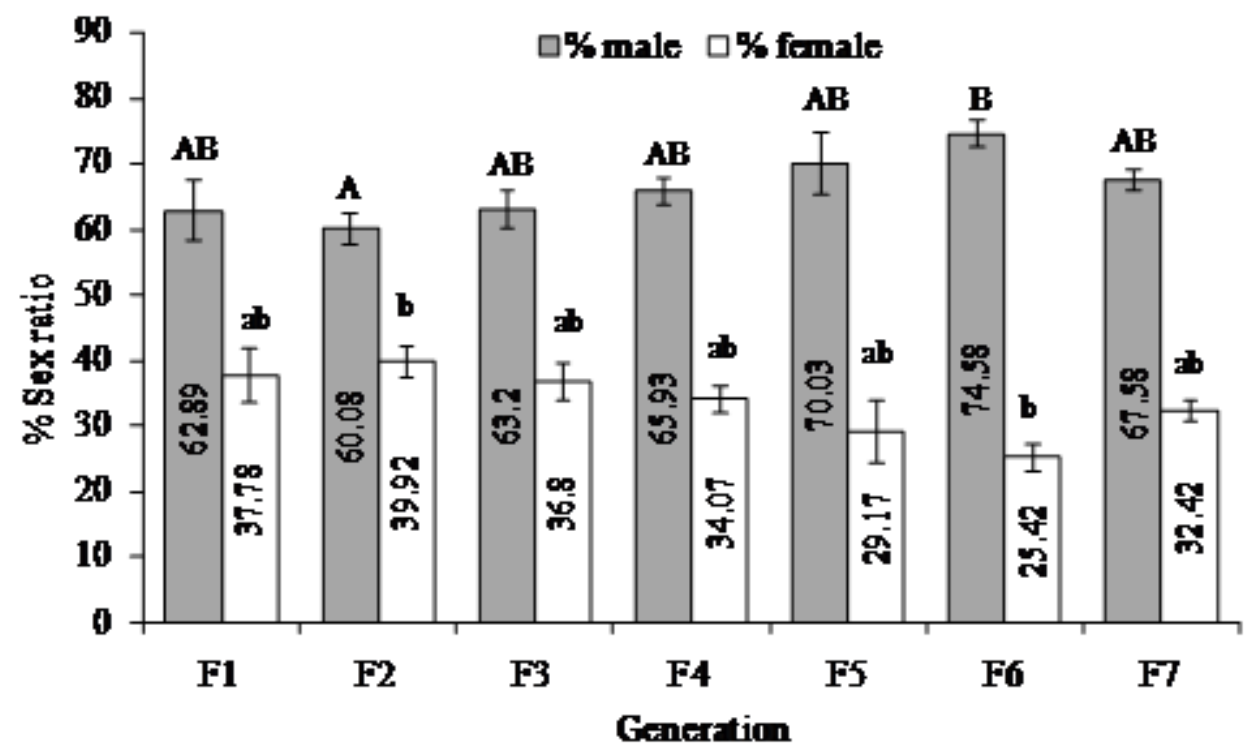

Figure 3. Adult progeny sex ratio (male, female) following sib mating (mother-son, brothersister crosses) in first generation in micro plot in the field and the successive generations in the laboratory. Bars followed by the same letters are not significantly different using Tukey,s HSD tests $(\mathrm{P}<0.05)$. Vertical line in bar show standard error of mean.

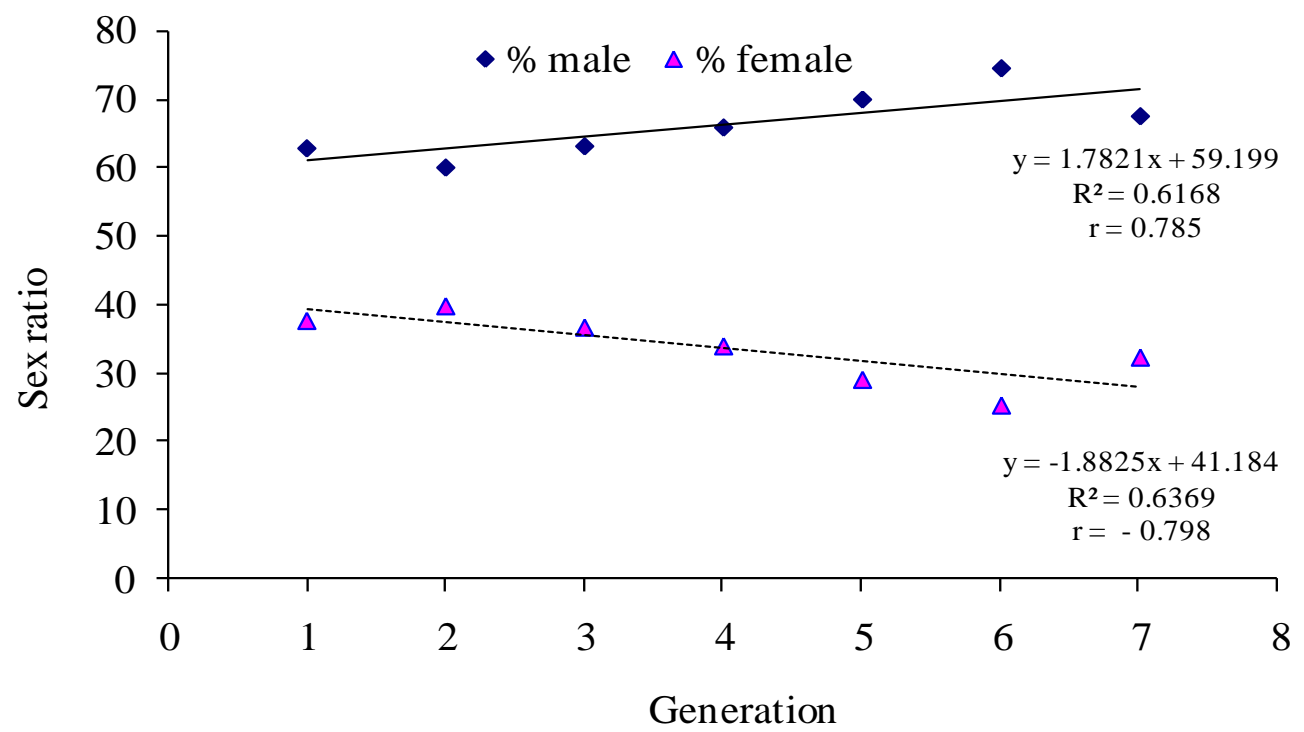

Figure 4. Relationship between sex ratio and generation following sib mating (mother-son, brother-sister crosses) in first generation in micro plot in the field and the successive generations in the laboratory. 


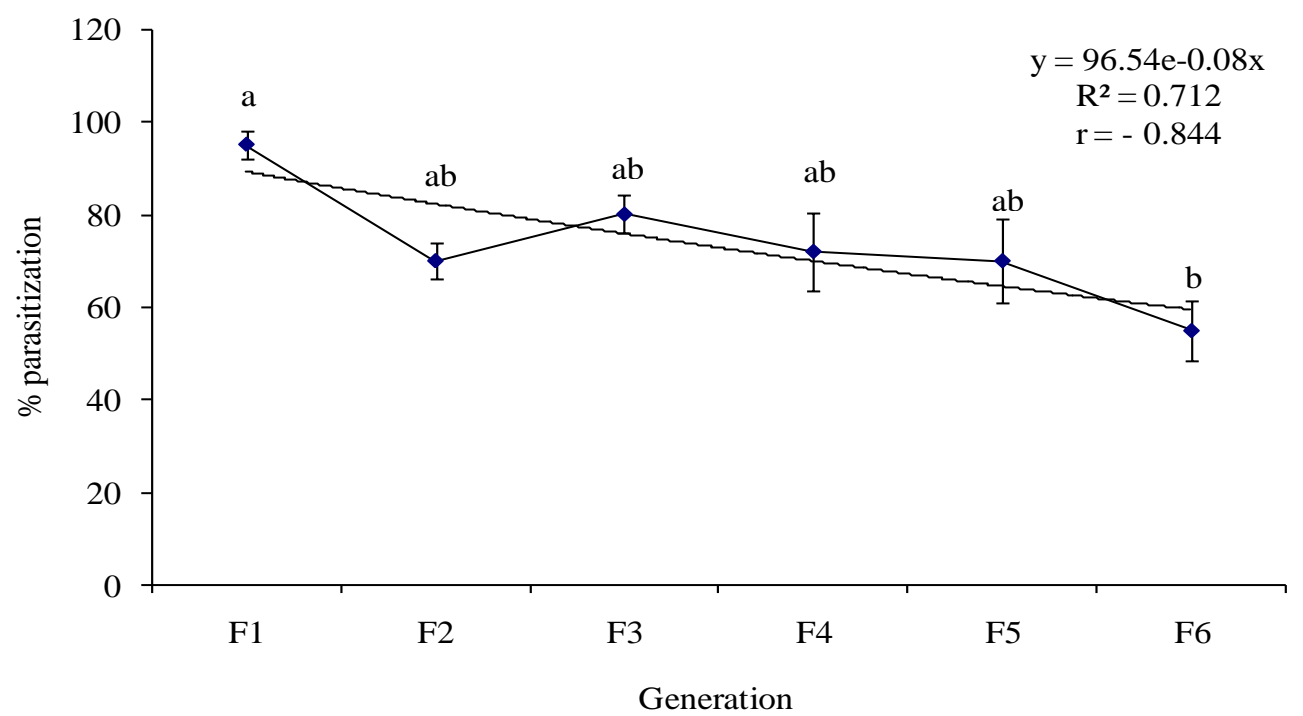

Figure 5. Generation wise mean parasirism by Bracon hebetor due to sib mating (mother-son, brother-sister crosses) through sixth generations in the first and successive generationcare in laboratory. Line followed by the same letters are not significantly different using Tukey,s HSD tests $(\mathrm{P}<0.05)$. Vertical line in line graph show standard error of mean.

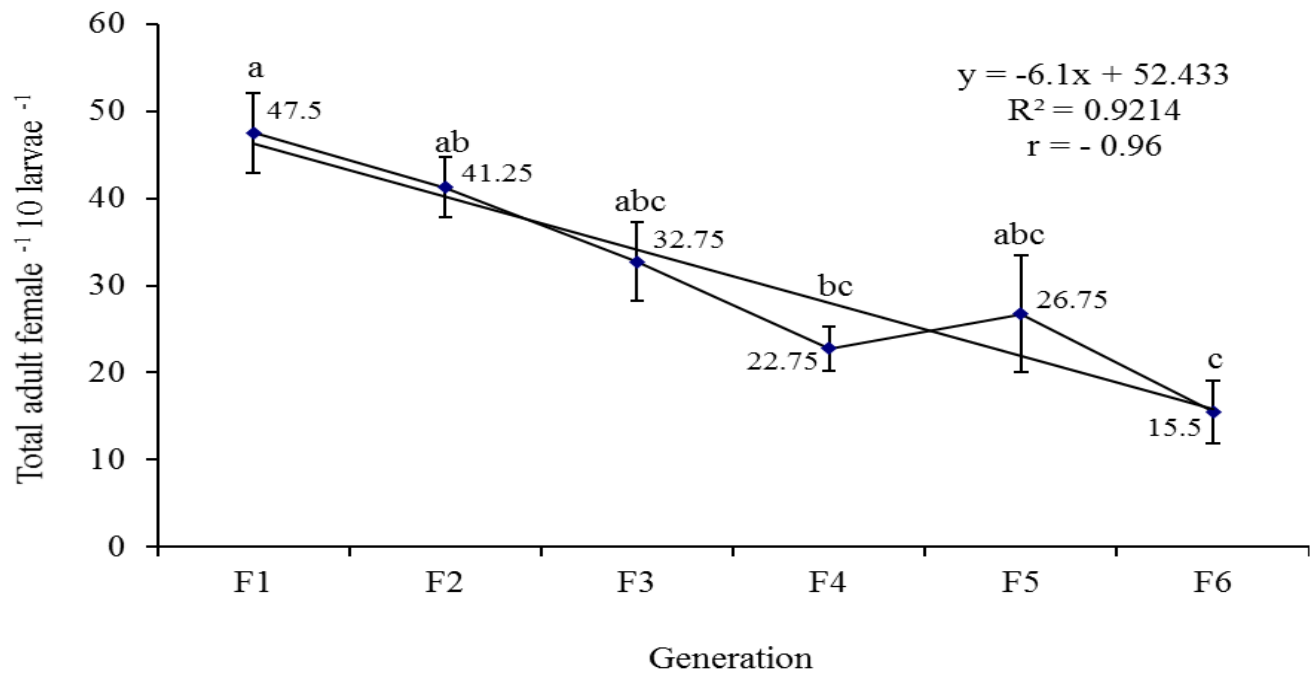

Figure 6. Generation wise total number of adult progeny Bracon hebetor produced per female Bracon hebetor following sib mating (mother-son, brother-sister crosses) from first to sixth generations in the laboratory. Line followed by the same letters are not significantly different using Tukey,s HSD tests $(\mathrm{P}<0.05)$. Vertical line in line graph show standard error of mean. 


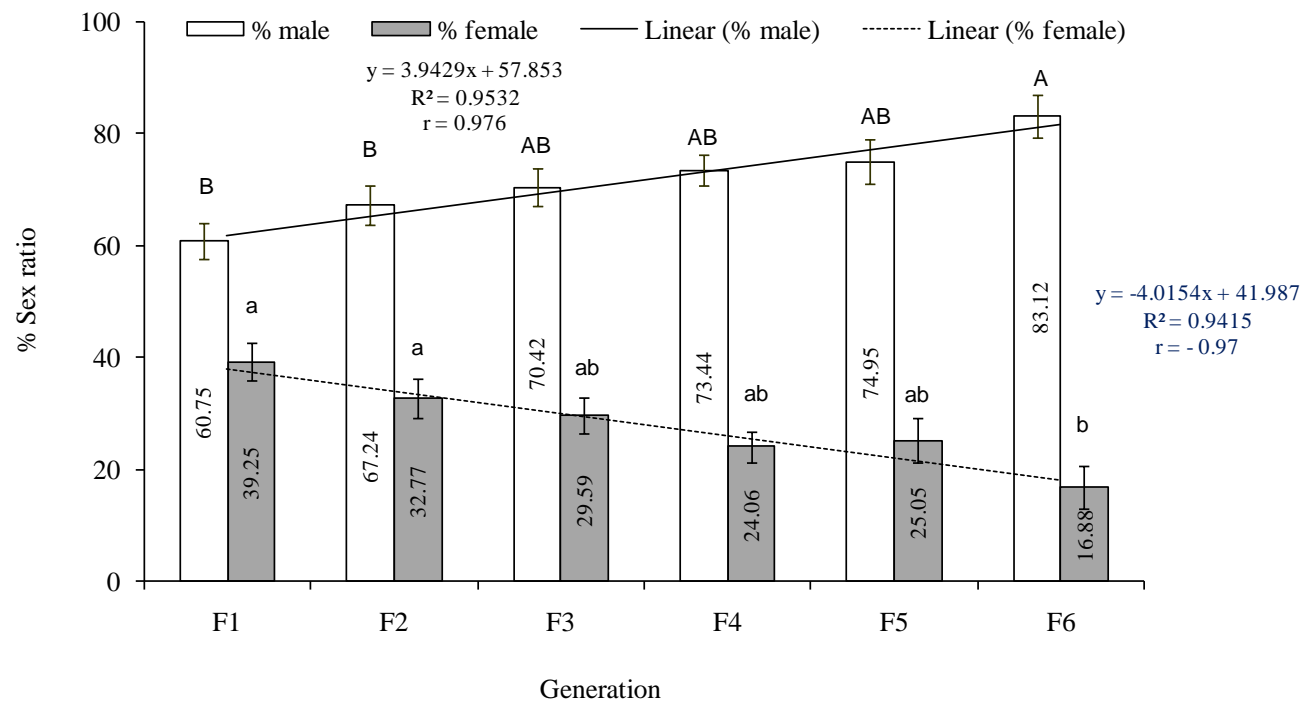

Figure 7. Male and female percentages of adult progeny of Bracon hebetor after sib mating (mother-son, brother-sister crosses) from first to sixth generations in laboratory.

Bars followed by the same letters are not significantly different using Tukey,s HSD tests $(\mathrm{P}<0.05)$. Vertical line in bar show standard error of mean. 